\title{
Phenformin inhibits cell proliferation and induces cell apoptosis and autophagy in cholangiocarcinoma
}

\author{
SHUYANG HU*, QING OUYANG ${ }^{*}$, QINGBAO CHENG ${ }^{*}$, JINGHAN WANG, FEILING FENG, \\ LIANG QIAO, WEI GAN, YANG SHI, DEMIN WU and XIAOQING JIANG \\ First Department of Biliary Surgery, Eastern Hepatobiliary Surgical Hospital, \\ The Second Military Medical University, Shanghai 200438, P.R. China
}

Received February 11, 2016; Accepted October 10, 2017

DOI: $10.3892 / \mathrm{mmr} .2018 .8573$

\begin{abstract}
Cholangiocarcinoma (CCA) is an aggressive malignant tumor and the prognosis of patients with advanced stage disease remains poor. Therefore, the identification of novel treatment agents for CCA is required. In the present study, the biological effects of the diabetes therapeutic agent, phenformin, in CCA cell lines was investigated. Cell Counting Kit-8 cell viability, cellular clone formation and subcutaneous tumor formation assays were performed, which revealed that phenformin inhibited CCA cell proliferation and growth both in vitro and in vivo. In addition, phenformin induced CCA cell apoptosis and autophagy. Phenformin partly activated the liver kinase B1 (LKB1)/5' AMP-activated protein kinase signaling pathway to exert its biological effects on CCA cell lines, as demonstrated by knockdown of LKB1, which reversed these effects. In conclusion, the present study demonstrated the biological effects of phenformin in CCA and suggested that phenformin may be a potential novel agent for CCA treatment.
\end{abstract}

\section{Introduction}

Cholangiocarcinoma (CCA) is a malignant tumor that arises from the epithelial cells in intrahepatic or extrahepatic bile ducts, and is difficult to diagnose and has a poor prognosis (1-3). Although surgical resection and liver transplantation are potentially curative treatments, the majority of patients present with unresectable tumors and succumb to disease within a year of diagnosis (4). CCA is frequently resistant to chemotherapy

Correspondence to: Professor Xiaoqing Jiang, First Department of Biliary Surgery, Eastern Hepatobiliary Surgical Hospital, The Second Military Medical University, 225 Shanghai Road, Shanghai 200438, P.R. China

E-mail: jxq1225@sina.com

${ }^{*}$ Contributed equally

Key words: cholangiocarcinoma, phenformin, cell proliferation, cell apoptosis, autophagy, liver kinase B1/5' AMP-activated protein kinase as results of monotherapy and combination chemotherapy are often disappointing (5-7). Therefore, finding novel chemical agents for the treatment of CCA is required.

The diabetes therapeutic biguanide compound, phenformin, has been demonstrated to inhibit complex I of the mitochondria (8). In previous studies, phenformin was identified to inhibit tumor growth in xenograft, transgenic and carcinogen-induced mouse cancer models $(9,10)$. As phenformin increases intracellular adenosine monophosphate (AMP) and adenosine diphosphate and subsequently induces the activation of liver kinase B1 (LKB1)/5' AMP-activated protein kinase(AMPK) signaling. The LKB1/AMPK axis may inhibit cancer cell proliferation and growth and functions as a tumor suppressive signaling pathway (8). Phenformin has the potential to be a novel agent that inhibits CCA cell proliferation and tumor growth.

In the present study, CCA cell lines were treated with phenformin and the biological effects were observed. Phenformin inhibited CCA cell proliferation and tumor growth and induced cell apoptosis and autophagy.

\section{Materials and methods}

Cell culture. BE and Huh28 human CCA cell lines were purchased from American Type Culture Collection (Manassas, VA, USA) and were maintained under standard culture conditions $\left(37^{\circ} \mathrm{C}\right.$ and $5 \% \mathrm{CO}_{2}$ ) in RPMI 1640 supplement with $10 \%$ (v/v) FBS (both Gibco; Thermo Fisher Scientific, Inc., Waltham, MA, USA).

RNA isolation and quantitative real-time polymerase chain reaction $(R T-q P C R)$. Total RNA was purified from CCA cells or CCA adjacent tissues using TRIzol (Invitrogen; Thermo Fisher Scientific, Inc.) according to the manufacturer's protocol. A total of $1 \mu \mathrm{g}$ RNA was reverse transcribed using SuperScript Reverse Transcriptase III (Invitrogen; Thermo Fisher Scientific, Inc.) in $37^{\circ} \mathrm{C}$ for $1 \mathrm{~h}$. RT-qPCR was performed using SYBR ${ }^{\circledR}$ Green Supermix (4473369; Applied Biosystems; Thermo Fisher Scientific, Inc.) on an Applied Biosystems 7500 PCR system with the following program: $95^{\circ} \mathrm{C}$ for $30 \mathrm{sec}$, followed by 40 cycles at $95^{\circ} \mathrm{C}$ for $5 \mathrm{sec}$ and $60^{\circ} \mathrm{C}$ for $31 \mathrm{sec}$, and finally $95^{\circ} \mathrm{C}$ for $15 \mathrm{sec}, 60^{\circ} \mathrm{C}$ for $1 \mathrm{~min}$ and $95^{\circ} \mathrm{C}$ for $15 \mathrm{sec}$. The relative expression of each gene was 
analyzed by the comparative cycle threshold method $\left(2^{-\Delta \Delta \mathrm{Cq}}\right.$ method) (11) which was normalized to GAPDH. Primers used in the present study are described in Table I.

Western blotting. Cells were lysed in IP lysis buffer (catalog no. P0013, Beyotime Institute of Biotechnology, Haimen, China), according to the manufacturer's protocol. Subsequently, the protein concentration was measured by BCA assay kit (Thermo Fisher Scientific, Inc.) and cell lysates were boiled in 5X SDS-PAGE loading buffer for $10 \mathrm{~min}$ and resolved by $8 \%$ SDS-PAGE prior to transfer onto a nitrocellulose membrane. The membrane was blocked by $5 \%(\mathrm{~m} / \mathrm{v})$ non-fat milk (BD Biosciences, Franklin Lakes, NJ, USA). The following primary antibodies were used: LKB1 (3047;1:1,000; Cell Signaling Technology, Inc., Danvers, MA, USA), phosphorylated (p)-AMPK (2532; 1:1,000; Cell Signaling Technology, Inc.), AMPK (2532; 1:1,000; Cell Signaling Technology, Inc.) and GAPDH (60004-1-Ig; 1:5,000; ProteinTech Group, Inc., Chicago, IL, USA). All these antibodies were incubated at $4^{\circ} \mathrm{C}$ overnight. Then the membranes were incubated with HRP-linked anti-rabbit IgG (7074; 1:2,000; Cell Signaling Technology, Inc.) or HRP-linked anti-mouse IgG (7076, 1:2,000; Cell Signaling Technology, Inc.) for $1 \mathrm{~h}$ at room temperature. Proteins were then visualized with an Enhanced Chemiluminescence kit (Thermo Fisher Scientific, Inc.).

Cell Counting Kit-8 (CCK-8) cell viability assays. Cells were seeded into a 96 -well plate at $3 \times 10^{3}$ cells per well with $100 \mu \mathrm{l}$ culture medium containing 0 or $2 \mathrm{mM}$ phenformin (Sigma-Aldrich; Merck KGaA, Darmstadt, Germany) and cultured at $37^{\circ} \mathrm{C}$ and $5 \% \mathrm{CO}_{2}$ for 12,24 or $48 \mathrm{~h}$. The cell viability was quantified by addition $10 \mu \mathrm{l}$ of CCK-8 reagent (Dojindo Molecular Technologies, Inc., Kumamoto, Japan). After a $1.5 \mathrm{~h}$ incubation, the optical density (OD) was measured using a Power Wave XS microplate reader (Omega Bio-Tek, Inc., Norcross, GA, USA) at a wavelength of $450 \mathrm{~nm}$.

Colony formation assays. A total of 500 cells/well were seeded in 6-well plates and incubated for 10 days at $37^{\circ} \mathrm{C}$ and $5 \% \mathrm{CO}_{2}$. Colonies were fixed and stained with $0.5 \%$ crystal violet at room temperature and the number of colonies was counted.

Flow cytometry cell apoptosis assays. A total of $2 \times 10^{5}$ cells/well were seeded into 6 -well plates and treated with 0 or $2 \mathrm{mM}$ phenformin in an incubator at $37^{\circ} \mathrm{C}$. After $24 \mathrm{~h}$, cells were washed twice with $1 \mathrm{X}$ cold PBS, and stained with Annexin V-fluorescein isothiocyanate (FITC)/propidium iodide (PI; BD Biosciences, Franklin Lakes, NJ, USA), according to the manufacture's protocol. BD FACSCalibur (BD Biosciences) was used for the detection and CellQuest Pro software (BD Biosciences) for the analysis.

In vivo tumor formation assay. A total of 10 male BALB/c (nu/nu) mice (Shanghai SLAC Laboratory Animal Co., Ltd.; 5 weeks old, mean weight $15 \mathrm{~g}$ ) were kept at $25^{\circ} \mathrm{C}$ and $45 \%$ humidity with 12-h light/dark cycle with free access to sterile food and water. Then, $4 \times 10^{6} \mathrm{RBE}$ cells were subcutaneously injected into the right flank of the mice. After 2 weeks, the mice were intratumorally injected with 0 (physiological saline) or $2 \mathrm{mM}$ phenformin (5 mice/group) every 4 days for
Table I. Sequences of primers and siRNAs.

\begin{tabular}{ll}
\hline Name & \multicolumn{1}{c}{ Sequence (5'-3') } \\
\hline BECN1 & F, TGGACACGAGTTTCAAGATCC \\
& R, CTCCTGGGTCTCTCCTGGTT \\
ATG5 & F, AAAGATGTGCTTCGAGATGTGT \\
& R, CACTTTGTCAGTTACCAACGTCA \\
ATG7 & F, ATGATCCCTGTAACTTAGCCCA \\
& R, CACGGAAGCAAACAACTTCAAC \\
GAPDH & F, AGCCTCAAGATCATCAGCAATGCC \\
siRNA-NC & R, TGTGGTCATGAGTCCTTCCACGAT \\
siRNA-LKB1 & ACGUGACACGUUCGGAGAATT \\
\hline
\end{tabular}

siRNA, short interfering RNA; BECN1, Beclin1 gene; ATG5, autophagy 5 gene; $A T G 7$, autophagy 7 gene; $\mathrm{NC}$, negative control; LKB1, liver kinase B1; F, forward; R, reverse.

A

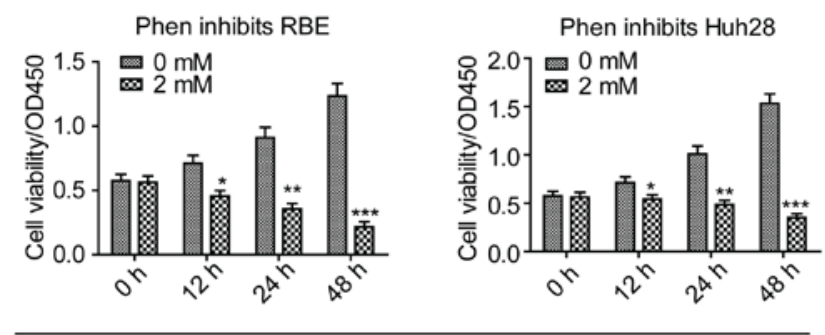

B
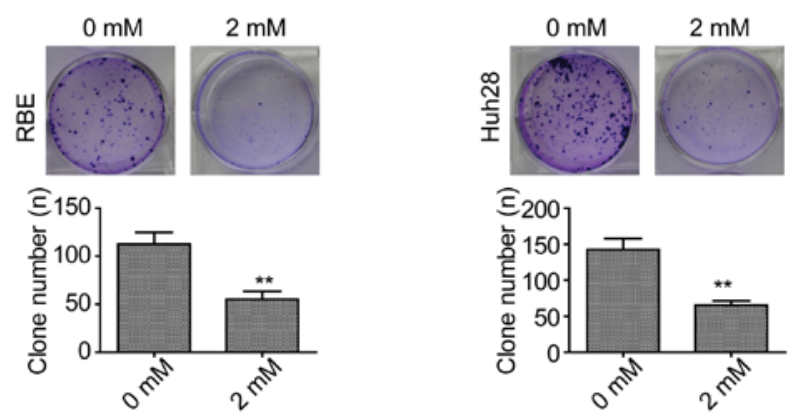

C
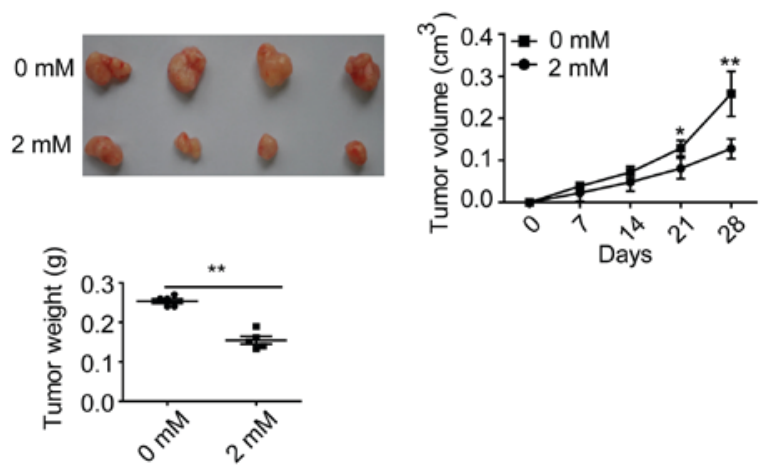

Figure 1. Phenformin inhibits CCA cell line proliferation and growth (A) Phenformin inhibits the proliferation of RBE and Huh28CCA cell lines. (B) Cellular clone formation assays indicated that phenformin inhibited RBE and Huh28 cell growth. (C) Subcutaneous tumor formation assays suggested that phenformin inhibited $\mathrm{RBE}$ cell proliferation and growth in vivo, $\mathrm{n}=5$. ${ }^{*} \mathrm{P}<0.05,{ }^{* *} \mathrm{P}<0.01$ and ${ }^{* * *} \mathrm{P}<0.00010 \mathrm{mM}$ phenformin. In vitro assays were performed in triplicate. CCA, cholangiocarcinoma; Phen, phenformin. 

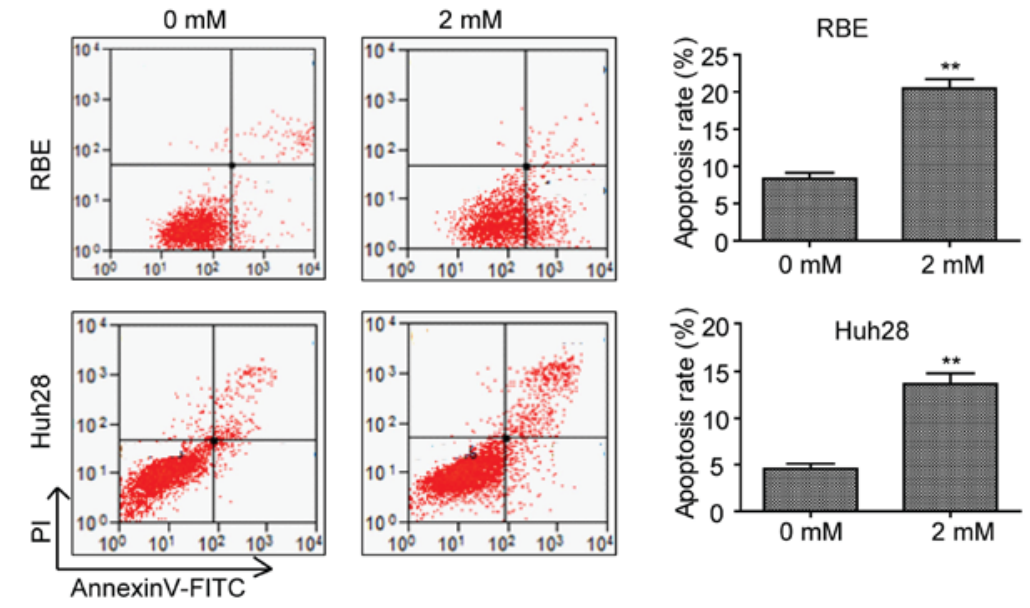

B
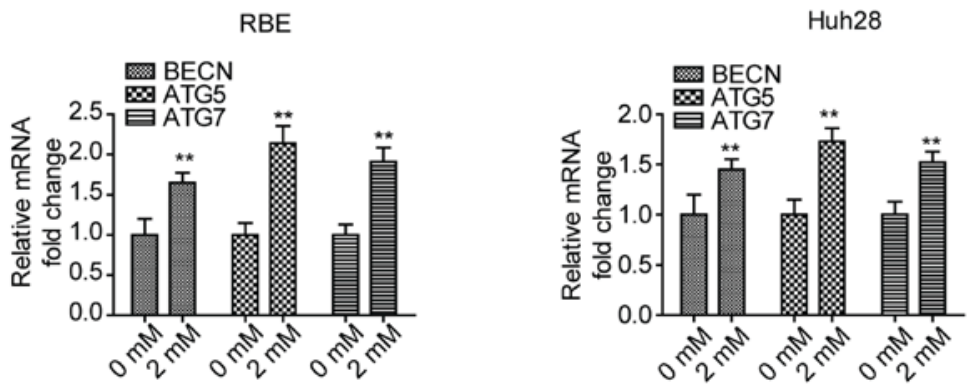

Figure 2. Phenformin promotes cholangiocarcinoma cell line apoptosis and autophagy. (A) Annexin V-FITC/PI cell apoptosis assays revealed that phenformin induces apoptosis in RBE and Huh28 cells. (B) mRNA expression levels of autophagy-associated genes, BECN1, ATG5 and ATG7, were elevated following $24 \mathrm{~h}$ phenformin treatment in RBE and Huh28 cells. ${ }^{* *} \mathrm{P}<0.01 \mathrm{vs} 0 \mathrm{mM}$ phenformin. Assays were performed in triplicate. FITC, fluorescein isothiocyanate; PI, propidium iodide; $B E C N 1$, Beclin1 gene; $A T G 5$, autophagy 5 gene; $A T G 7$, autophagy 7 gene.

a month. Tumor sizes were measured once a week and mice were sacrificed for the analysis of tumor burden after 4 weeks. The procedures in the present study were approved by the Ethics Committee of the Second Military Medical University (Shanghai, China).

siRNA treatment. The sequences for LKB1 siRNAs are described in Table I. A total of $3 \times 10^{5}$ RBE and Huh28 cells were transfected with $100 \mathrm{nM}$ LKB1 siRNA or with $100 \mathrm{nM}$ negative control siRNA (Table I) using Lipofectamine ${ }^{\circledR} 2000$ (Invitrogen; Thermo Fisher Scientific, Inc.). After $24 \mathrm{~h}$ these transfected cell lines were used to perform the subsequent assays.

Statistical analysis. Data are expressed as the mean \pm standard deviation; two-way analysis of variance followed by the LSD post-hoc test was performed for comparisons between groups. GraphPad Prism 5 (GraphPad Software, Inc., La Jolla, CA, USA) was used for analysis. $\mathrm{P}<0.05$ was considered to indicate a statistically significant difference.

\section{Results}

Phenformin inhibits CCA cell line proliferation and growth in vitro and in vivo. An inhibitor of mitochondrial complex 1 , phenformin, has been demonstrated to inhibit tumor growth in lung cancer and delay tumor progression in a lymphoma mouse model (8). In the present study, CCK-8 assays were performed after treating two CCA cell lines, RBE and Huh28, with $2 \mathrm{mM}$ phenformin. Phenformin significantly inhibited the proliferation of these cell lines compared with cells treated with $0 \mathrm{mM}$, after 12, 24 and $48 \mathrm{~h}$ (Fig. 1A). Clone formation assays revealed that phenformin significantly inhibited the ability of the cell lines to form clones compared with cells treated with $0 \mathrm{mM}$ phenformin $(\mathrm{P}<0.01$; Fig. 1B). Subcutaneous tumor formation assays revealed that mice injected with $2 \mathrm{mM}$ phenformin exhibited a significantly smaller RBE tumor volume compared with the $0 \mathrm{mM}$ control group after 28 days $(\mathrm{P}<0.01$; Fig. $1 \mathrm{C})$. These results suggested that phenformin inhibits CCA cell proliferation and growth in vitro and in vivo.

Phenformin induces CCA cell apoptosis and autophagy. As phenformin inhibited cell proliferation, the role of phenformin in CCA cell apoptosis and autophagy was determined. When cells were treated with $2 \mathrm{mM}$ phenformin for $24 \mathrm{~h}$, a significant amount of cell death was observed compared with cells treated with $0 \mathrm{mM}$ phenformin $(\mathrm{P}<0.01$; Fig. $2 \mathrm{~A})$. In addition, the mRNA expression levels of autophagy-associated genes, Beclin-1 (BECN1), autophagy (ATG)5 and ATG7 (12) were upregulated after treatment with $2 \mathrm{mM}$ phenformin in the two cell lines compared with cells treated with $0 \mathrm{mM}$ phenformin (Fig. 2B). These findings suggested that phenformin induces CCA cell apoptosis and autophagy. 
A

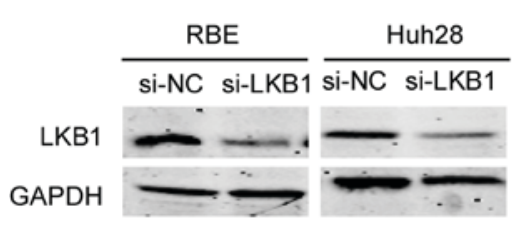

B

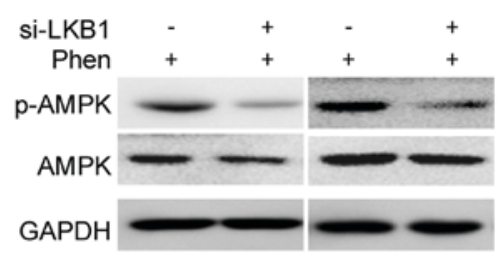

C

RBE si-LKB1/Phenoformin

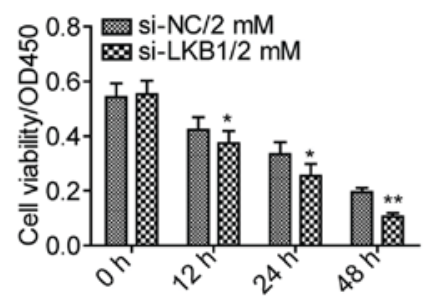

Huh28 si-LKB1/Phenformin

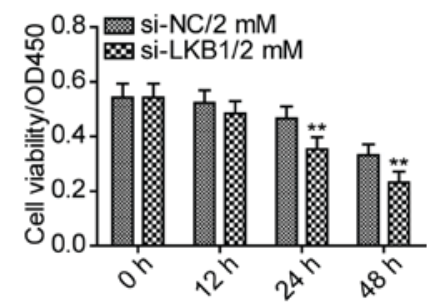

D
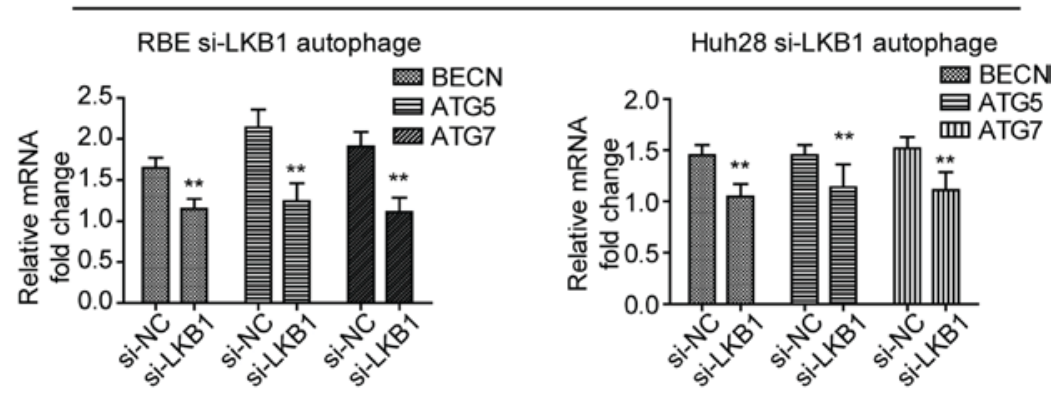

Figure 3. Phenformin exerts its effects via the LKB1/AMPK axis in cholangiocarcinoma cell lines. (A) Protein expression levels of LKB1 were reduced following transfection of cells with an LKB1-specific siRNA. (B) Knockdown of LKB1 inhibited the activation and phosphorylation of AMPK in the two cell lines following treatment with $2 \mathrm{mM}$ phenformin. (C) Knockdown LKB1 significantly inhibited the cell viability of the two cell lines. (D) mRNA expression levels of autophagy-associated genes, BECN1, ATG5 and ATG7, were reduced following knockdown of LKB1 in cell lines treated with phenformin for $24 \mathrm{~h}$. Assays were performed in triplicate. ${ }^{*} \mathrm{P}<0.05$ and ${ }^{* *} \mathrm{P}<0.01$ vs. siRNA-NC. NC, negative control; BECN1, Beclin1 gene; ATG5, autophagy 5 gene; ATG7, autophagy 7 gene; LKB1, liver kinase B1; AMPK, 5 ' AMP-activated protein kinase; Phen, phenformin; siRNA; short interfering RNA.

Phenformin exerts its effects via the LKBI/AMPK axis in CCA. A previous study revealed that phenformin induces apoptosis in LKB1-deficient non-small cell lung cancer cell lines (13). This finding suggests that phenformin may activate the LKB1/AMPK signaling pathway to inhibit CCA cell proliferation and induce apoptosis and autophagy. In the present study, the protein expression levels of LKB1 was reduced in RBE and Huh28 cell lines following knockdown of LKB1 using a specific siRNA, compared with cells treated with negative control siRNA (Fig. 3A). The expression levels of the target of LKB1, p-AMPK, following knockdown of LKB1 in the cell lines was measured after treatment with phenformin. Knockdown of LKB1 reduced the expression levels of p-AMPK when treated with phenformin for $12 \mathrm{~h}$ (Fig. 3B). In addition, the cell viability was reduced in LKB1 knockdown cells with phenformin treatment, as determined by a CCK-8 cell viability assay (Fig. 3C). The mRNA expression levels of autophagy-associated genes, BECN1, ATG5 and ATG7, were reduced in LKB1 knockdown cell lines treated with phenformin, compared with cells transfected with negative control siRNA $(\mathrm{P}<0.01$; Fig. 3D). These results suggested that phenformin exerts its effects via activation of the LKB1/AMPK signaling pathway.

\section{Discussion}

The present study investigated the biological effects of phenformin in CCA cell lines and demonstrated that phenformin inhibited CCA cell proliferation and growth and induced cell apoptosis and autophagy.

Phenformin is a biguanide compound and paralog to metformin, which is widely used in as a therapy for type 2 diabetes worldwide (14). Phenformin has been previously demonstrated to exhibit potential anti-cancer properties. Shackelford et al (13) revealed that phenformin inhibits LKB1-deficient non-small cell lung cancer progression. Liu et al (15) reported that phenformin induces cell cycle alterations and apoptosis in breast cancer. Cancer cells exhibit alterations in cellular metabolism to enable their continued growth and proliferation $(16,17)$. Phenformin is a biguanide compound that inhibits complex 1 of mitochondria, resulting in increased intracellular AMP and ADP that induces the LKB1/AMPK signaling loop (8). AMPK is a conserved sensor of intracellular energy levels (18) and phenformin treatment and the activation of the LKB1/AMPK signaling pathway may significantly inhibit anabolic pathways and cell proliferation (19). In the present study, phenformin significantly 
inhibited CCA cell proliferation and induced cell apoptosis and autophagy. These effects may be induced by the LKB1/AMPK signaling pathway in CCA cell lines.

Wang et al (20) reported that LKB1 is frequently downregulated in CCA tissues. The findings of the present study suggested that silencing of LKB1 in combination with phenformin led to these cells being more susceptible to death. The current findings suggest that Phenformin has the potential to be a therapeutic agent for CCA treatment.

\section{Acknowledgements}

The present study was funded by The National Natural Science Fund (grant no. 81472280).

\section{References}

1. Bismuth H, Nakache $\mathrm{R}$ and Diamond T: Management strategies in resection for hilar cholangiocarcinoma. Ann Surg 215: 31-38, 1992.

2. Lim J and Park C: Pathology of cholangiocarcinoma. Abdom Imaging 29: 540-547, 2004.

3. Khan SA, Toledano MB and Taylor-Robinson SD: Epidemiology, risk factors, and pathogenesis of cholangiocarcinoma. $\mathrm{HPB}$ (Oxford) 10: 77-82, 2008 .

4. Khan SA, Davidson BR, Goldin R, Pereira SP, Rosenberg WM, Taylor-Robinson SD, Thillainayagam AV, Thomas HC, Thursz MR and Wasan H; British Society of Gastroenterology: Guidelines for the diagnosis and treatment of cholangiocarcinoma: Consensus document. Gut 51 (Suppl 6): VI1-VI9, 2002.

5. Anderson CD, Pinson CW, Berlin J and Chari RS: Diagnosis and treatment of cholangiocarcinoma. Oncologist 9: 43-57, 2004.

6. Khan SA, Davidson BR, Goldin RD, Heaton N, Karani J, Pereira SP, Rosenberg WM, Tait P, Taylor-Robinson SD, Thillainayagam AV, et al: Guidelines for the diagnosis and treatment of cholangiocarcinoma: An update. Gut 61: 1657-1669, 2012.

7. Knol JA and Pelletier SJ: Primary Carcinomas of the Liver. In: Surgical treatment: Resection and transplantation. Cambridge University Press, Cambridge, pp177-194, 2009.
8. Hawley SA, Ross FA, Chevtzoff C, Green KA, Evans A, Fogarty S, Towler MC, Brown LJ, Ogunbayo OA, Evans AM and Hardie DG: Use of cells expressing $\gamma$ subunit variants to identify diverse mechanisms of AMPK activation. Cell Metab 11: 554-565, 2010.

9. Algire C, Amrein L, Bazile M, David S, Zakikhani M and Pollak M: Diet and tumor LKB1 expression interact to determine sensitivity to anti-neoplastic effects of metformin in vivo. Oncogene 30: 1174-1182, 2011.

10. Memmott RM, Mercado JR, Maier CR, Kawabata S, Fox SD and Dennis PA: Metformin prevents tobacco carcinogen-induced lung tumorigenesis. Cancer Prev Res (Phila) 3: 1066-1076, 2010.

11. Livak KJ and Schmittgen TD: Analysis of relative gene expression data using real-time quantitative PCR and the 2(-Delta Delta C(T)) method. Methods 25: 402-408, 2001.

12. Pyo JO, Yoo SM and Jung YK: The interplay between autophagy and aging. Diabates Metab J 37: 333-339, 2013

13. Shackelford DB, Abt E, Gerken L, Vasquez DS, Seki A, Leblanc M, Wei L, Fishbein MC, Czernin J, Mischel PS and Shaw RJ: LKB1 inactivation dictates therapeutic response of non-small cell lung cancer to the metabolism drug phenformin. Cancer Cell 23: 143-158, 2013.

14. Davidson MB and Peters AL: An overview of metformin in the treatment of type 2 diabetes mellitus. Am J Med 102: 99-110, 1997.

15. Liu Z, Ren L, Liu C, Xia T, Zha X and Wang S: Phenformin induces cell cycle change, apoptosis, and mesenchymal-epithelial transition and regulates the AMPK/mTOR/p70s6k and MAPK/ERK pathways in breast cancer cells. PloS One 10: e0131207, 2015.

16. Muñoz-Pinedo C, El Mjiyad N and Ricci JE: Cancer metabolism: Current perspectives and future directions. Cell Death Dis 3: e248, 2012.

17. Kroemer G and Pouyssegur J: Tumor cell metabolism: Cancer's Achilles' heel. Cancer Cell 13: 472-482, 2008.

18. Shaw RJ: Glucose metabolism and cancer. Curr Opin Cell Biol 18: 598-608, 2006.

19. Shackelford DB and Shaw RJ: The LKB1-AMPK pathway: Metabolism and growth control in tumour suppression. Nat Rev Cancer 9: 563-575, 2009.

20. Wang J, Zhang K, Wang J, Wu X, Liu X, Li B, Zhu Y, Yu Y, Cheng Q, Hu Z, et al: Underexpression of LKB1 tumor suppressor is associated with enhanced Wnt signaling and malignant characteristics of human intrahepatic cholangiocarcinoma. Oncotarget 6: 18905-18920, 2015. 